\title{
TROLLEYOLOGY: ¿DE QUIÉN ES EL DILEMA DEL TRANVÍA?
}

TROLLEYOLOGY: WHOSE TROLLEY DILEMMA IS IT?

\author{
Fabio Morandin-Ahuerma \\ Profesor Investigador de tiempo completo \\ Benemérita Universidad Autónoma de Puebla \\ Complejo Regional Nororiental \\ Fabio.morandin@correo.buap.mx \\ México, Puebla
}

\section{SUMARIO}

- Introducción

- Philippa Ruth Foot

- Judth Jarvis Thomson

- Hans Welzel

- Karl Engisch

- Análisis

- Conclusiones

\section{RESUMEN}

El autor recobra las fuentes originales del llamado Dilema del tranvía pues considera que existe confusión sobre quién es el autor original. Sostiene que no es Phillipa Foot como suele citarse comúnmente, ni siquiera Judith Thomson, sino que sus raíces son más lejanas y se encuentran en dos juristas alemanes: Hans Welzel y, aún antes, Karl Engisch. Propone que la solución al dilema está dada desde el Derecho positivo y no en especulaciones consecuencialistas.

\section{ABSTRACT}

The author recovers the original sources of the so-called Trolley Dilemma because he considers that there is confusion about who the original author is. He argues that it is not Phillipa Foot as it is commonly cited, not even Judith Thomson, but that its roots are more distant and are found in two German jurists: Hans Welzel and, even earlier, Karl Engisch. Proposes that the solution to the dilemma is given from the positive law and not in consequentialist speculations.

\section{PALABRAS CLAVE}

Dilema del tranvía; teoría del doble efecto; daño colateral; teoría causalista; teoría de la acción finalista.

\section{KEYWORDS}

Trolley dilemma; double effect theory; collateral damage; causalist theory; finalist action theory.

\section{INTRODUCCIÓN}

Supongamos que un tranvía sin frenos se desplaza rápidamente. Más adelante hay cinco personas atadas a la vía principal sobre su curso actual. Hay un ramal que podría desviarlo hacia otra vía, sin embargo, hay otra persona atada a esa vía. ¿Se debería mover la palanca para salvar la vida de esas cinco personas, a costa de la vida de una? [Versión libre].

Este artículo pone de manifiesto que prevalecen dos confusiones respecto a la autoría del llamado Dilema del tranvía (DT). La primera surge de creer que la filósofa británica, Phillippa Foot fue quien planteó el DT tal como se presenta actualmente, mientras que ella, únicamente, dibujó una referencia indirecta al problema. Sin embargo, es frecuentemente que sea citada como la autora original. En cambio, quien construyó el dilema actual, le dio su nombre y ofreció variables del mismo fue la filósofa norteamericana, Judith Thomson. Sin 
embargo, surge una segunda confusión, y es que ni Foot ni Thomson plantearon el primer escenario dilemático de trenes o tranvías. El primer antecedente se encuentra en el jurista alemán, Karl Engisch quien hizo una primera alusión al postrero DT; después, el también jurista alemán, Hans Welzel planteará un problema dilemático similar. El punto aquí es que ni Foot, ni Thomson, ni otros autores posteriores (Petrinovich \& ONeill, 1996; Greene et al., 2001; Kamm, 2015) por citar algunos, se refirieron a los trabajos de Engisch (1930) ni al de Welzel (1951) quienes, de alguna manera, habían resuelto dicho problema desde el marco normativo de lo estrictamente legal. El propósito del presente trabajo, no es resolver el DT sino, únicamente, analizar qué fue literalmente lo que cada uno de los autores dijo, y de qué manera el DT debería ser resuelto, más allá de un supuesto enfrentamiento de posturas morales entre deontologistas y consecuencialistas.

\section{PHILIPPA RUTH FOOT (1920-2010)}

Suele citarse —más de mil 800 veces, según Google Scholar - a la filósofa inglesa Philippa R. Foot como la autora original del DT. En 1967 planteó una serie de escenarios dilemáticos en un artículo titulado The problem of abortion and the doctrine of double effect [El problema del aborto y la doctrina del doble efecto] en el que analizó las implicaciones que las decisiones con implicaciones morales pueden tener en otras personas. Cuáles son las consecuencias directas, indirectas de una acción moral y, la diferencia, por ejemplo, entre matar y dejar morir.

En el artículo The problem of abortion... que se publicó en la revista The Oxford Review, Foot planteó varios aspectos y llamó la atención sobre las consecuencias que un acto puede ocasionar, ya sea de manera voluntaria o involuntaria. También abordó los imperativos éticos que subyacen al hacer algo o simplemente, no hacer algo, esto es, dejar que las cosas sucedan. La diferencia entre actuar o permitir, matar o dejar que mueran y la hipotética obligación moral de proporcionar ayuda a quien la necesita o al menos, no causar daño a los demás.

El trabajo de Foot (1967), como ella misma explica, fue una respuesta a otro artículo publicado por el filósofo del derecho, Herbert
Lionel Adolphus Hart, titulado Intention and Punishment (1967) [Intención y castigo], quien distinguía los conceptos de culpabilidad e inocencia, tratando de pasar por encima de los debates filosóficos sobre el determinismo y el libre albedrío.

El escenario dilemático presentado por Foot (1967,pág. 8), y cuya cita sacaré del contexto, dice así:

(1) (...) To make the parallel as close as possible it may rather be supposed that he is the driver of a runaway tram, which he can only steer from one narrow track on to another; five men are working on one track and one man on the other; anyone on the track he enters is bound to be killed.

[Traducción libre: Para hacer un paralelo se puede suponer que él es el conductor de un tranvía fuera de control que solo puede llevarlo de una estrecha vía a la otra; cinco hombres están trabajando en una y un solo hombre en la otra; cualquiera que esté en el camino morirá].

Ella se refería, como veremos a continuación, al piloto de un avión que está a punto de caer $y$ debe decidir si hacer el aterrizaje forzoso en una zona poblada o en una zona menos poblada. Por lo anterior, siendo el texto más amplio, creo que es necesario citar el pasaje completo en el que Foot (1967, pág. 8) se refirió tangencialmente al hoy denominado DT:

(2) Suppose that a judge or magistrate is faced with rioters demanding that a culprit be found for a certain crime and threatening otherwise to take their own bloody revenge on a particular section of the community. The real culprit being unknown, the judge sees himself as able to prevent the bloodshed only by framing some innocent person and having him executed. Beside this example is placed another in which a pilot whose airplane is about to crash is deciding whether to steer from a more to a less inhabited area. To make the parallel as close as possible it may rather be supposed that he is the driver of a runaway tram which he can only steer from one narrow track on to another; five men are working on one track and one man on the other; anyone on the track he enters is bound to be killed. In the case of the riots the mob have five hostages, so that in both the exchange is supposed to be one man's life for the lives of five. The question is why we should say, without hesitation, that the driver should steer for the less occupied track, while most of us would be appalled at the idea that the innocent man could be framed. It may 
be suggested that the special feature of the latter case is that it involves the corruption of justice, and this is, of course, very important indeed. But if we remove that special feature, supposing that some private individual is to kill an innocent person and pass him off as the criminal we still find ourselves horrified by the idea. The doctrine of double effect offers us a way out of the difficulty, insisting that it is one thing to steer towards someone foreseeing that you will kill him and another to aim at his death as part of your plan.

[Traducción libre: Supongamos que un juez o magistrado se enfrenta a alborotadores que exigen que se encuentre a un culpable de un determinado delito y que amenazan con tomar una sangrienta venganza en contra un sector particular de la comunidad. Al no saber quién es el verdadero culpable, el juez se ve a sí mismo como capaz de prevenir el derramamiento de sangre solo al formularle cargos a una persona inocente y ejecutarlo. Además de este ejemplo, se pone otro en el que un piloto cuyo avión está a punto de colapsarse decide si debe dirigirse desde un área más habitada a una zona menos habitada. Para hacer un paralelo se puede suponer que él es el conductor de un tranvía fuera de control que solo puede llevarlo de una estrecha vía a la otra; cinco hombres están trabajando en una y un solo hombre en la otra; cualquiera que esté en el camino morirá. En el caso de los disturbios, la turba tiene a cinco rehenes, por lo que, en ambos casos, se supone que el intercambio debe ser la vida de un hombre por la vida de cinco. La pregunta es ¿por qué deberíamos decir, sin dudarlo, que el conductor debe dirigirse a la pista menos ocupada, mientras que la mayoría de nosotros estaría horrorizada ante la idea de que el hombre inocente podría ser ejecutado? Se puede sugerir que la característica especial de este último caso es que implica la corrupción de la justicia, y esto es, por supuesto, muy importante. Pero si eliminamos esa característica especial, suponiendo que alguien mate a una persona inocente y lo haga pasar por delincuente, aún nos horroriza esa idea. La doctrina del doble efecto nos ofrece una manera de salir de esa dificultad, insistiendo en que una cosa es dirigirse hacia alguien previendo que morirá (intención oblicua), y otra es que se dirija su muerte como parte del plan (intención directa)].

Foot sólo quería analizar si era lícito o no salvar la vida de un mayor número de personas, causándole la muerte a otros. El llamado daño colateral que, por ejemplo, en escenarios militares queda justificado
(USAF, 1998) pero que filosóficamente tiene mayores implicaciones que una interpretación meramente utilitarista. En el caso dilemático del juez, Foot se refiere a un sector de la comunidad al que se podría salvar, condenando a un inocente, por un delito que no cometió. Y, en el caso de los rehenes y de los trabajadores ferroviarios es nuevamente el dilema moral de salvar la vida de cinco personas a costa de la muerte de una.

Finalmente, Foot dijo que no es lícito salvar la vida de unos, matando a personas inocentes. Criticó la doctrina del doble efecto (DDE) y señaló que, una cosa es que muera alguien de manera accidental, como un daño colateral oblicuo, y otra cosa es que exista la intención de hacerle daño a alguien deliberadamente, así sea por una causa presumiblemente justa.

En el caso del aborto que analizó, supuso que la muerte del feto es inminente, ya sea porque se trate de un aborto profiláctico, por ejemplo, para salvar la vida de la madre; o ya sea deliberadamente por no querer asumir las responsabilidades inherentes a la maternidad; el resultado siempre será el mismo: por lo regular, el aplastamiento del cráneo para poder extirpar al producto, lo cual, sin importar las causas o la finalidad, siempre será mortal para la futura vida.

\section{JUDITH JARVIS THOMSON (N. 1929)}

Posteriormente la filósofa estadounidense Judith Jarvis Thomson prestó mayor atención (1976; 1985) a los casos hipotéticos que Foot había planteado, y escribió sus propias versiones de algunos de los dilemas de la autora inglesa.

La versión del DT que Thomson planteó en 1976 en su artículo Killing, letting die, and the trolley problem [Matar, dejar morir, y el problema del tranvía] fue la siguiente:

(3) Edward is the driver of a trolley, whose brakes have just failed. On the track ahead of him are five people; the banks are so steep that they will not be able to get off the track in time. The track has a spur leading off to the right, and Edward can turn the trolley onto it. Unfortunately there is one person on the right-hand track. Edward can turn the trolley, killing the one; or he can refrain from turning the trolley, killing the five. (Thomson, 1976, pág. 206). 
[Traducción libre: Edward es el conductor de un carro, cuyos frenos acaban de fallar. En la vía, más adelante, hay cinco personas; Las orillas son tan empinadas que no podrán salir de la pista a tiempo. La pista tiene un ramal que conduce a la derecha, y Edward puede girar el tranvía hacia ella. Desafortunadamente hay una persona en la vía de la derecha. Edward puede hacer girar al tranvía, matando a uno; o puede abstenerse de girarlo, matando a los cinco].

Más adelante, en el mismo trabajo, Thomson introduce nuevos elementos al dilema tales como: "All six are innocent people whom villains have tied to the trolley tracks, five on one track, one on the other" (1976, pág. 211) [Los seis son personas inocentes que los villanos han atado a las pistas de tranvía, cinco en una pista, uno en el otro].

Años después, Thomson publicó en mayo de 1985 otro artículo titulado: The trolley problem [El problema del tranvía] en la revista The Yale Law Journal, en el que hizo una nueva versión del DT y, añadió otras perspectivas al problema de la intencionalidad directa e indirecta.

El giro teórico que planteó, y que es determinante para la discusión de los mecanismos de toma de decisión, es que en lugar se proyectar al participante como conductor del tren, esto es Edward; lo externalizó como alguien que está a un lado de la vía, al que llamó Bystander at The Switch [testigo en el cambio de agujas] quien igualmente tiene la posibilidad de desviar el tranvía hacia uno u otro lado:

(4) Let us now consider a case I will call Bystander at the Switch. In that case you have been strolling by the trolley track, and you can see the situation at a glance: The driver saw the five on the track ahead, he stamped on the brakes, the brakes failed, so he fainted. What to do? Well, here is the switch, which you can throw, thereby turning the trolley yourself. Of course, you will kill one if you do. But I should think you may turn it all the same (Thomson, 1985, pág. 1397).

[Traducción libre: Permítasenos considerar ahora un caso al que llamaré el testigo en el cambio de agujas. En este escenario, haz estado paseando por la pista del tranvía, y observas la situación de un vistazo: el conductor del tranvía vio a los cinco más adelante en la pista, pisó el freno, los frenos fallaron, por lo que se desmayó.
¿Qué hacer? Bueno, aquí está el cambio de agujas, que puedes activar haciendo que gire el tranvía. Por supuesto que matarás a uno si lo haces. Pero pienso que el resultado final será el mismo (matarás a alguien)].

Thomson introdujo variantes que serán relevantes para las discusiones posteriores. Agregó una nueva perspectiva al personalizar o despersonalizar al conductor. No es lo mismo que sea Edward quien conduce, a que sea un testigo anónimo que se encuentre fuera del tren, esto es, es diferente el abordaje siendo el analista quien se ve a sí mismo como conductor o suponer que sería otro quien lo haga. Observar es ser espectador, en cambio conducir es ser protagonista.

En una versión se refiere a un paseante, un testigo impersonal, alguien que ve cómo el tranvía se precipita contra las personas. Hay un interruptor o cambio de agujas [switch] con el que se puede variar el curso de la acción.

Thomson además implantó un nuevo dilema al que denominó Dilema de la pasarela o Dilema del puente, conocido también como Fat. Ahora, nuevamente el participante entra en el conflicto de salvar a cinco personas a costa de la vida de un inocente; Sin embargo, en esta ocasión, el inocente es un hombre gordo sobre un puente que, si es empujado hacia abajo sobre las vías, detendrá con su cuerpo la marcha del tranvía y salvará la vida de las cinco personas amarradas. Un dilema de Thomson, por demás hipotético, pero que pretende demostrar que quienes estaban de acuerdo en mover el curso del tranvía en testigo en el cambio de agujas, ahora no están tan seguros de su decisión, a pesar de que debería ser el mismo cálculo: salvar cinco vidas a costa de sacrificar a una.

(5) Consider a case-which I shall call Fat Man in which you are standing on a footbridge over the trolley track. You can see a trolley hurtling down the track, out of control. You turn around to see where the trolley is headed, and there are five workmen on the track where it exits from under the footbridge. What to do? Being an expert on trolleys, you know of one certain way to stop an out-of-control trolley: Drop a really heavy weight in its path. But where to find one? It just so happens that standing next to you on the footbridge is a fat man, a really fat man. He is leaning over the railing, watching the trolley; all you have to do is to give him a little shove, and over the railing 
he will go, onto the track in the path of the trolley. Would it be permissible for you to do this? (Thomson 1985, pág. 1410).

[Traducción libre: Considere un caso, que llamaré Hombre Gordo, en el que usted está parado en un puente peatonal sobre la vía del tranvía. Usted ve al tranvía que se abalanza por la vía, fuera de control. Usted se da la vuelta para ver hacia dónde se dirige el tranvía, y hay cinco obreros abajo en los rieles a la salida del puente peatonal. ¿Qué hacer? Como es un experto en tranvías, usted sabe que hay una forma de detener que vaya fuera de control: dejar caer un peso muy pesado en su camino. ¿Pero dónde encontrar uno? Da la casualidad de que, parado junto a usted en el puente peatonal, hay un hombre gordo, un hombre realmente gordo. Él se inclina sobre la barandilla, mirando el carro; todo lo que tiene usted que hacer es darle un pequeño empujón, y romperá el barandal, cayendo a los rieles en el camino del tranvía. ¿Sería permisible para usted hacer esto?].

Como se puede observar, el método de salvar a las cinco personas cambió. Ya no se trata de oprimir un botón o mover una palanca hacia una vía donde está la otra persona; aquí es el participante quien debe, por su propia mano, hacer contacto con la persona obesa y empujarlo para que caiga a las vías del tranvía $\mathrm{y}$, supuestamente, detenga su paso. Según Thomson (1985) esta variable hace que quienes estaban de acuerdo en desviar el tranvía, cambien de opinión o no estén tan seguros de continuar apoyando un criterio utilitarista para salvar a los cinco.

\section{HANS WELZEL (1904-1977)}

Hans Welzel, jurista alemán, en su artículo Zum Notstandsproblem [El problema de la emergencia] publicado en la revista Zeitschrift für die gesamte Strafrechtswissenschaft [Revista de Derecho penal] hizo, antes que Thomson y antes que Foot, una reflexión alusiva al DT. El dilema que presentó fue el siguiente:

(6) Ein Güterzug droht wegen falscher Weichenstellung auf einen vollbesetzten stehenden Personenzug aufzufahren. Ein Weichensteller erkennt die Gefahr und leitet den Güterzug auf ein Nebengleis um, so dass dieser in eine Gruppe von Gleisarbeitern rast, die alle zu Tode kommen. Wie ist die Strafbarkeit des

\begin{abstract}
Weichenstellers zu beurteilen? (Welzel 1951, pág. 50).
\end{abstract}

[Traducción libre: Un tren de carga amenaza con chocar contra un tren de pasajeros debido a que el primero ha tomado una dirección equivocada. Un guardavía reconoce el peligro y desvía el tren de carga hacia una pista lateral, de modo que embiste a un grupo de trabajadores que están ahí, y todos ellos mueren. ¿Cómo evaluar la responsabilidad penal del guardavía?]

El punto dilemático para Welzel fue la responsabilidad que debe o debería imputársele al trabajador ferroviario como resultado de su acción, dado que se generó un daño, al parecer no visualizado. El tren arrolló y mató a quienes estaban trabajando en la vía y, sin embargo, evitó que chocara el tren de carga contra el tren de pasajeros.

Por eso, considera que las normas jurídicas deben ir más allá del mero acto específico y su intencionalidad, si bien la finalidad es relevante, Welzel (1951) se decanta por los resultados que dicha acción ocasiona. La doctrina de la acción se concentra entonces en el hecho de entrar en un escenario en el que se obtiene un resultado y, este, por supuesto, produce un efecto, más la imputabilidad de la acción está en los efectos, no en el designio.

Así fue como Welzel construyó un ámbito de interpretación jurídico distinto al que se había discutido hasta entonces; la teoría de la acción finalista se enfrenta con la teoría causalista porque pone énfasis en el resultado o fin de una acción que se persigue libremente, esto es, por voluntad propia del sujeto, independientemente de cuáles podrían haber sido las causas que movieron al sujeto a actuar de esa manera. La construcción de causas de la acción puede ser, en general, múltiple pues cada individuo encuentra una serie de posibles causas para su acción, especialmente si se trata de un hecho delictivo por lo que, en algunos casos, se llega a desplegar excusas inverosímiles como causa que motiva al delito (Welzel, 1961).

\section{KARL ENGISCH (1899-1990)}

Sin embargo, el primer antecedente del DT se encuentra en la tesis del jurista alemán Karl Engisch (1930) titulada Untersuchungen über Vorsatz und Fahrlässigkeit im Strafrecht [Investigaciones sobre dolo e imprudencia en 
el Derecho penal] cuyo texto presentó para habilitarse como catedrático de la Universidad de Friburgo. En su trabajo textualmente afirma:

(6) Es kann sein, daß ein Weichensteller, um einen drohenden Zusammenstoß zu verhindern, der aller Vorsicht nach sehr viel Menschenleben kosten wird, den Zug so leitet, daß zwar auch Menschenleben aufs Spiel gesetzt werden, aber sehr viel weniger, als wenn er den Dingen ihren Lauf ließe (Engisch, 1930, pág. 288).

[Traducción libre: Puede ser que un guardagujas para evitar una colisión inminente que, con toda probabilidad, costará una gran cantidad de vidas humanas, dirija el tren hacia donde también haya vidas humanas y que se pongan también en riesgo, pero muchas menos que si hubiera seguido el tren su curso].

El jurista aborda el problema de la irrupción del curso de una acción que tendría una repercusión y que, en cambio, en caso de evitarse, cambiaría su rumbo y, por tanto, su efecto. El modelo de compensación de Engisch consiste en sopesar cuál es el propósito que se persigue, y si los efectos del curso de una acción son preferibles a la intervención para obtener otro efecto con mayor valía (Engisch, 1930). El foco está en la infracción de un mandato legal, si acaso queda o no justificado por el resultado deseado, esto es, los alcances del rompimiento de la ley y los resultados positivos que se esperan, pero violentando el marco normativo establecido (Zurkinden, 2016).

\section{ANÁLISIS}

Volveré a los planteamientos originales de Engisch y de Welzel, y a lo que la teoría causal establece: Una acción es producto de la determinación de un sujeto de entrar en movimiento, esto es, quien actúa y produce un efecto. Sin importar cuál es la intención que la persona pueda tener para mover el elemento $\mathrm{A}$, a la posición $\mathrm{B}$, el hecho es que efectúa una acción que hace que un estado de cosas en el mundo cambie. Cuál es la intención que pueda tener esa persona para mover algo de su entorno, para Welzel (1951), es algo secundario, lo mismo para Engisch (1930). Lo principal es el efecto que causa A sobre $\mathrm{C}$ al moverse a $B$. Una acción es punible siempre y cuando la persona que comete el acto tenga la voluntad de hacerlo, no importa sus intenciones, simplemente quiere hacerlo, y lo hace. Existe la causa, el nexo causal y el efecto de la acción.

El nexo causal se refiere a la conexión que existe entre un acto y el daño que produce. Es la causa y el efecto que establece cuál es exactamente el hecho, la acción, que determinó o produjo ese daño; repito, qué es lo que causa ese efecto y es ahí donde puede determinarse la culpabilidad del responsable o quien determinó realizar esa acción (Bustamante, 1993).

Desde esa óptica, el guardavía de Welzel ha visto que el tren de carga embestirá al tren de pasajeros y decide cambiar su curso, tal vez sin advertir o advirtiendo que en la pista adyacente hay trabajadores ferroviarios, los cuales, por efecto directo o indirecto, pierden la vida.

La pregunta que considero relevante y que hace el propio Welzel es: ¿Se le debe juzgar por haber matado a los trabajadores o se debe considerar como un daño colateral al efecto benéfico de salvar de un choque contra el vagón de pasajeros? La respuesta causalista que hasta hoy se sigue discutiendo es si el guardavías es culpable de la muerte de los trabajadores y, por tanto, debe pagar por ello. El derecho positivo responderá que: Sí.

Por tanto, tampoco los dilemas presentados por Thomson $(1976,1985)$ deberían ofrecer mayor resistencia para su solución. Cualquiera que actúe deliberadamente y su acción conlleve la muerte de inocentes, así sea con las mejores intenciones, será juzgado por homicidio, y de acuerdo a las circunstancias es que se le dará mayor o menos imputabilidad por sus agravantes.

Si se piensa en la vida real y se imagina alguien que va caminando por una vía en donde hay cinco trabajadores en los rieles que no pueden escuchar que un tren se acerca rápidamente por el ruido que producen unas perforadoras hidráulicas junto a ellos $\mathrm{y}$, si se decidiera mover las agujas hacia una vía donde se hay un solo trabajador, que tampoco escucha el tren venir, seguramente habría culpa para quien decide intervenir por su propio fuero y tratara de salvar a los cinco trabajadores. No importa porqué lo haga, el Juez dirá que el efecto de la acción produjo una persona muerta, lo demás, podría ser secundario. 
Sin embargo, no es mi objetivo en este trabajo analizar la pertinencia o no del DT, ni sus profundas implicaciones filosóficas entre el utilitarismo y el deontologismo. El objetivo ha sido demostrar que no se le ha hecho justicia académica, ni a Karl Engisch ni a Hans Welzel como los autores originales del Dilema del tranvía, al menos la idea de la cual se desarrolla. Si bien es Judith Thomson quien les da la forma actual inspirada por Phillippa Foot, ni Foot, ni Thomson citan alguna referencia a Engisch o a Welzel a quienes, especulo, debieron haber leído.

\section{CONCLUSIÓN}

Aunque suele citarse a Foot (1967) como la autora original del DT, fue Thomson (1976; 1985) quien le dio forma actual al dilema; Sin embargo, sostengo que ambas pudieron haber sido inspiradas por los planteamientos dilemáticos hechos muchos años atrás por Welzel (1951) así como por Engish (1930) pero no fueron citados por ninguna de las dos (al menos en los trabajos de 1967; 1976 y 1985), como tampoco son citados, o tal vez ni siquiera conocidos, por autores posteriores que suelen considerar que el DT es autoría de Phillppa Foot (Cfr. Petrinovich \& ONeill, 1996; Greene et al. 2001; Kamm, 2015).

No es mi intención resolver definitivamente el DT, seguirá por muchos años discutiéndose entre filósofos y psicólogos, pero considero que deben aclararse los términos prácticos, de la vida real, en que aplican los sistemas penales actuales. En ellos se juzga la acción, el hecho y sus consecuencias, más allá de la motivación intrínseca que lleva al sujeto para actuar en consecuencia.

\section{FUENTES DE INFORMACIÓN}

\section{Fuentes bibliográficas}

Bustamante, J. (1993). Teoría general de la responsabilidad civil. Buenos Aires: AbeledoPerrot.

Engisch, K. (1930/1964). Untersuchungen über Vorsatz und Fahrlässigkeit im Strafrecht. Berlin: Aalen Scientia-Verl.

\section{Fuentes electrónicas}

Foot, P. (1967). The problem of abortion and the doctrine of double effect. Oxford Review 5, 5-15. DOI: 10.1093/0199252866.003.0002. Disponible en: http://www.oxfordscholarship. com/view/10.1093/0199252866.001.0001/ acprof-9780199252862-chapter-2

Greene, J., Sommerville, R.B., Nystrom, L., Darley, J.M., \& Cohen, J.D. (2001). An fMRI investigation of emotional engagement in moral judgment. Science, 293(5537), 2105-2108. DOI: $10.1126 /$ science. 1062872 . Disponible en: https://www.ncbi.nlm.nih.gov/ pubmed/11557895

Hart, H. L. A. (1967/2009). Intention and Punishment. Oxford: Oxford University Press. Disponible en: http://www. oxfordscholarship.com/view/10.1093/acp rof:oso/9780199534777.001.0001/acprof9780199534777-chapter-5

Kamm, F. M. (2015). The trolley problem mysteries. Oxford: Oxford University Press. Disponible en: http://www. oxfordscholarship.com/view/10.1093/ acprof:oso/9780190247157.001.0001/ acprof-9780190247157

Petrinovich, L., \& O'Neill, P. (1996). Influence of wording and framing effects on moral intuitions. Ethology and Sociobiology, 17(3), 145-171. Disponible en: https:// www.sciencedirect.com/science/article/ pii/0162309596000416

Thomson, J. (1976). Killing, letting die, and the trolley problem. The Monist, 59(2), 204-217. https://doi.org/10.5840/monist197659224.

Thomson, J. (1985). The Trolley Problem. The Yale Law Journal, 94(6), 1395-1415. DOI: 10.2307/796133. Disponible en: https:// digitalcommons.law.yale.edu/cgi/viewcontent. cgi?referer=https://www.google.com/\&httpsre $\operatorname{dir}=1 \&$ article $=6930 \&$ context $=y l j$

USAF (1998). USAF Intelligence Targeting Guide. Air Force Pamphlet 14-210 Intelligence. Disponible en: https://fas.org/irp/doddir/usaf/ afpam14-210/part20.htm 
Welzel, H. (1951). Zum Notstandsproblem. Zeitschrift für die gesamte Strafrechtswissenschaft 63, 47-56. doi: 10.1515/zstw.1951.63.1.47. Disponible en: https://www.degruyter.com/view/j/ zstw.1951.63.issue-1/zstw.1951.63.1.47/ zstw.1951.63.1.47.xml
Welzel, H. (1961/2011). El nuevo sistema del Derecho penal: Una introducción a la doctrina de la acción finalista. Montevideo: B de F.

Zurkinden, N. (2016). Crash beim Testbetrieb selbstfahrender Fahrzeuge. Jusletter IT (24). Disponible en: https://jusletter-it.weblaw.ch/ issues/2016/24-November-2016/crash-beimtestbetri_91fd98526d.html\#footnote_50 